\title{
Optical and electronic properties of low-density InAs/lnP quantum-dot-like structures designed for single-photon emitters at telecom wavelengths
}

Holewa, P.; Gaweczyk, M.; Ciostek, C.; Wyborski, P.; Kadkhodazadeh, Shima; Semenova, Elizaveta; Syperek, M.

Published in:

Physical Review B

Link to article, DOI:

10.1103/PhysRevB.101.195304

Publication date:

2020

Document Version

Publisher's PDF, also known as Version of record

Link back to DTU Orbit

Citation (APA):

Holewa, P., Gaweczyk, M., Ciostek, C., Wyborski, P., Kadkhodazadeh, S., Semenova, E., \& Syperek, M. (2020). Optical and electronic properties of low-density InAs/InP quantum-dot-like structures designed for single-photon emitters at telecom wavelengths. Physical Review B, 101(19), [195304].

https://doi.org/10.1103/PhysRevB.101.195304

\section{General rights}

Copyright and moral rights for the publications made accessible in the public portal are retained by the authors and/or other copyright owners and it is a condition of accessing publications that users recognise and abide by the legal requirements associated with these rights.

- Users may download and print one copy of any publication from the public portal for the purpose of private study or research.

- You may not further distribute the material or use it for any profit-making activity or commercial gain

- You may freely distribute the URL identifying the publication in the public portal 


\title{
Optical and electronic properties of low-density InAs/InP quantum-dot-like structures designed for single-photon emitters at telecom wavelengths
}

\author{
P. Holewa $\odot,{ }^{1}$ M. Gawełczyk $\odot,{ }^{2,1, *}$ C. Ciostek $\odot,{ }^{1, \dagger}$ P. Wyborski $\odot,{ }^{1}$ S. Kadkhodazadeh $\odot,{ }^{3}$ \\ E. Semenova $\odot,{ }^{4, \ddagger}$ and M. Syperek $\circledast^{1, \S}$ \\ ${ }^{1}$ Laboratory for Optical Spectroscopy of Nanostructures, Department of Experimental Physics, \\ Wroctaw University of Science and Technology, Wybrzeże Wyspiańskiego 27, 50-370 Wrocław, Poland \\ ${ }^{2}$ Department of Theoretical Physics, Wrocław University of Science and Technology, 50-370 Wroctaw, Poland \\ ${ }^{3}$ DTU Nanolab-National Centre for Nano Fabrication and Characterization, Technical University of Denmark, \\ Kongens Lyngby DK-2800, Denmark \\ ${ }^{4}$ DTU Fotonik, Technical University of Denmark, Kongens Lyngby DK-2800, Denmark
}

(Received 16 December 2019; revised manuscript received 27 February 2020; accepted 18 March 2020; published 11 May 2020)

\begin{abstract}
Due to their band-structure and optical properties, InAs/InP quantum dots (QDs) constitute a promising system for single-photon generation at the third telecom window of silica fibers and for applications in quantum communication networks. However, obtaining the necessary low in-plane density of emitters remains a challenge. Such structures are also still less explored than their InAs/GaAs counterparts regarding optical properties of confined carriers. Here, we report on the growth via metal-organic vapor phase epitaxy and investigation of low-density InAs/InP QD-like structures, emitting in the range of 1.2-1.7 $\mu \mathrm{m}$, which includes the $\mathrm{S}, \mathrm{C}$, and $\mathrm{L}$ bands of the third optical window. We observe multiple photoluminescence (PL) peaks originating from flat QDs with the height of a few material monolayers. Temperature-dependent PL reveals a redistribution of carriers between families of QDs. Via time-resolved PL, we obtain radiative lifetimes nearly independent of emission energy in contrast to previous reports on InAs/InP QDs, which we attribute to strongly height-dependent electron-hole correlations. Additionally, we observe neutral and charged exciton emission from spatially isolated emitters. Using the eight-band $\boldsymbol{k} \cdot \boldsymbol{p}$ model and configuration-interaction method, we successfully reproduce the energies of emission lines, the dispersion of exciton lifetimes, the carrier activation energies, as well as the biexciton binding energy, which allows for a detailed and comprehensive analysis of the underlying physics.
\end{abstract}

DOI: 10.1103/PhysRevB.101.195304

\section{INTRODUCTION}

Semiconductor epitaxial self-assembled quantum dots (QDs) have attracted attention for decades. Among many factors, this interest is driven by their ability to emit single photons of high purity and indistinguishability [1-3], and by the possibilities of their integration with existing semiconductor platforms. The proposed applications require further development of QDs to meet specific requirements, e.g., emission wavelength or spatial isolation of an emitter. A currently vital example is a nonclassical single photon source desired for quantum-secured data transfer and quantum information processing in open systems exploiting the existing architecture of mid- and long-haul silica- fiber-based optical networks. To achieve this specific application target, a QD should emit in the low-loss third spectral window of silica fibers $(1.46-1.625 \mu \mathrm{m})$ commonly divided into three subbands: S (1460-1530 nm), C (1530-1565 nm), and

\footnotetext{
*michal.gawelczyk@pwr.edu.pl

${ }^{\dagger}$ Present address: Experimental Physics 3, University of Würzburg, Am Hubland, 97074 Würzburg, Germany.

‡esem@fotonik.dtu.dk

§marcin.syperek@pwr.edu.pl
}

L (1565-1625 nm). This can be reached by employing an InGaAs metamorphic layer approach for the well-known InAs/GaAs QD structure [4]. Recently, QDs made by this technique have shown high fidelity photon entanglement [5]. However, this growth method is technologically demanding and still prone to structural defects [6], which obstructs its migration to the realm of applications.

Emission in the third telecom window can naturally be achieved by using InAs QDs grown on the InP substrate without applying additional strain engineering to the band gap. However, due to more than two times smaller lattice mismatch in comparison to standard InAs/GaAs QDs, the InAs/InP dots form dense arrays of flat and sizable in-plane material islands. Consequently, the in-plane spatial isolation and quality of such dots are hardly achievable but still indispensable for a single-photon source application.

Up to now, the reduced planar density of InAs/InP QDs emitting in the targeted spectral range has been achieved in molecular beam epitaxy (MBE) through modifications of the growth scheme or post-growth annealing of the structure [7-9]. A similar approach has been used in metal-organic vapor phase epitaxy (MOVPE), where the combination of annealing with double-capping was applied to enhance the uniformity of QD sizes [10,11]. Both techniques have yielded good optical quality InAs/InP QD-based emitters $[8,12,13]$. 
Here, we explore the MOVPE growth of InAs/InP QD-like nanostructures, modified by introducing the additional interruption step leading to formation of sparse islands emitting light within the third telecom band. The strain-driven formation of islands (the Stranski-Krastanov mechanism) from the $\operatorname{InAs}(\mathrm{P})$ layer is partially suppressed by high $\mathrm{V} / \mathrm{III}$ ratio to ensure low surface density of QDs below $10^{9} \mathrm{~cm}^{-2}$. This results in good spatial isolation of single emitters allowing for examination of their optical properties.

Apart from their relevance for applications, InAs QDs grown on InP itself or in alloys lattice-matched to InP, e.g., InGaAlAs with various cation proportions, are interesting for fundamental research, as they provide carrier confinement distinct from the one known from well-explored and described GaAs-based systems. Recent studies have provided explanation of some of their properties, such as partially polarized emission [14], exciton recombination characterized by two distinct lifetimes in the case of asymmetric dots [15] or the structure of excited states [16]. They have also raised a question regarding the exciton confinement regime $[17,18]$. These results were, however, obtained mostly for very dense ensembles of elongated QDs [19], and thus characterization of carrier confinement and the resulting optical properties of low-density QDs, growing in a different geometry such as those considered here, is needed. In this work, we combine both goals and provide a comprehensive description of this specific low-density QD-like system, focusing both on its relevance for potential applications and on fundamental properties of carrier confinement leading to the observed properties of emission.

The investigated InAs/InP QDs are formed on a 3-MLthick $\operatorname{InAs}(\mathrm{P})$ wetting layer (WL) and have a truncated pyramidal shape with a hexagonal base. The ensemble photoluminescence (PL) from the dots is concentrated in multiple narrow bands corresponding to QDs with height of consecutive integer numbers of monolayers (MLs) of material, according to our calculation. By fine-tuning the agreement of calculated exciton energies with positions of PL peaks, we establish that QDs in-plane size scales linearly with their height. Additionally, from time-resolved PL studies, we assess exciton lifetimes on the order of $1 \mathrm{~ns}$ with a very weak dispersion of values. While in general a dispersive character of the latter is expected due to strong scaling of radiative recombination rates with emission wavelength [20], we find theoretically that the virtually energy-independent values measured here result from the interplay between the said general trend and a strong change in the strength of Coulomb electron-hole correlations, known to enhance exciton-light coupling, with increasing QD height. We observe also a thermally driven redistribution of carriers, exhibited in thermal quenching of PL from the highest-energy peaks and a combination of enhancement and quenching for the others. For the latter, the extracted activation energies correspond well to those calculated by us for escape of electrons to the WL, while for the other we rather deal with excitation of whole excitons. At the same time, the energies corresponding to PL enhancement for each of the bands correspond to energies of thermal escape of single carriers from QDs forming the higher-energy neighboring band, which illustrates the migration of carriers between families of QDs. Finally, by means of high-resolution microphotoluminescence $(\mu \mathrm{PL})$ measurements performed on samples with fabricated submicrometer mesas, we observe emission lines originating from recombination of neutral and charged excitonic complexes within single QDs. Charged excitons (CX) are observed in each of the subbands of the third telecom window with emission persisting up to $T=70 \mathrm{~K}$.

The paper is organized as follows. In Sec. II, we describe the investigated structure and experimental methods. Next, in Sec. III, we describe the formalism employed for theoretical calculations. In Sec. IV, we report experimental results and provide explanations based on theoretical calculations. Finally, we summarize our findings in Sec. V.

\section{INVESTIGATED STRUCTURE AND OPTICAL EXPERIMENTS}

In this section, we discuss the QD growth method, and the morphological characteristics of the structures under consideration, as well as we describe the experimental methods used.

\section{A. Investigated structure}

The structure was grown in the low-pressure MOVPE TurboDisc ${ }^{\circledR}$ reactor using standard precursors: arsine $\left(\mathrm{AsH}_{3}\right)$, phosphine $\left(\mathrm{PH}_{3}\right)$, tertiarybutylphosphine (TBP), and trimethylindium (TMI). The growth sequence started from the deposition of a $0.5-\mu \mathrm{m}$-thick InP buffer layer on an (001)oriented InP substrate at $600^{\circ} \mathrm{C}$. Subsequently, the temperature was decreased to $480^{\circ} \mathrm{C}$ and stabilized first under TBP and afterwards $\mathrm{AsH}_{3}$ ambient. A layer of QDs on a wetting layer (WL) was formed after deposition of a nominally 1.04ML-thick InAs layer at TMIn and $\mathrm{AsH}_{3}$ flow rates of 12.2 and $4461 \mu \mathrm{mol} / \mathrm{min}$, respectively, with the $\mathrm{V} / \mathrm{III}$ ratio of 366. After that, a 6-s-long growth interruption was introduced prior to the deposition of a 10-nm-thick InP cap layer. The temperature was then increased to $600^{\circ} \mathrm{C}$, and $30 \mathrm{~nm}$ of $\mathrm{InP}$ was deposited. Finally, an array of surface QDs was deposited using the same growth parameters for characterization by atomic force microscopy (AFM).

The structural and morphological characteristics of the structures are summarized in Fig. 1 and further elaborated in Ref. [21]. Figure 1(a) shows a $5 \mu \mathrm{m} \times 3.5 \mu \mathrm{m}$ areal scan of surface QDs measured with the AFM. The dots are relatively large and slightly elongated in the [1/10] direction and are sparsely scattered with a surface density of $\lesssim 10^{9} \mathrm{~cm}^{-2}$. Statistical analysis of their geometry presented in Fig. 1(c) showed length $L$ in the range of 55-85 nm, while width $W$ is spread between 45 and $55 \mathrm{~nm}$. This gives an average in-plane aspect ratio of $\sim 1.25$. The median QD height $H$ is relatively large and exceeds $9 \mathrm{~nm}$. Setting the aspect ratio of $L / W=1.25$, we calculate $L$ from measured QD areas and check their correlation with $H$ by plotting in Fig. 1(d) the dependence $H(L)$ in the form of a scatter plot. Based on this we deduce a linear dependence between $H$ and $L$ for surface QDs, which is plotted to guide the eye with a solid line.

The magnified image of surface QDs shown in the inset of Fig. 1(a) reveals their diamond-like shape ended from top by a dome, which we schematically present in Fig. 1(e). Although these dots are optically inactive, we assume that their in-plane 

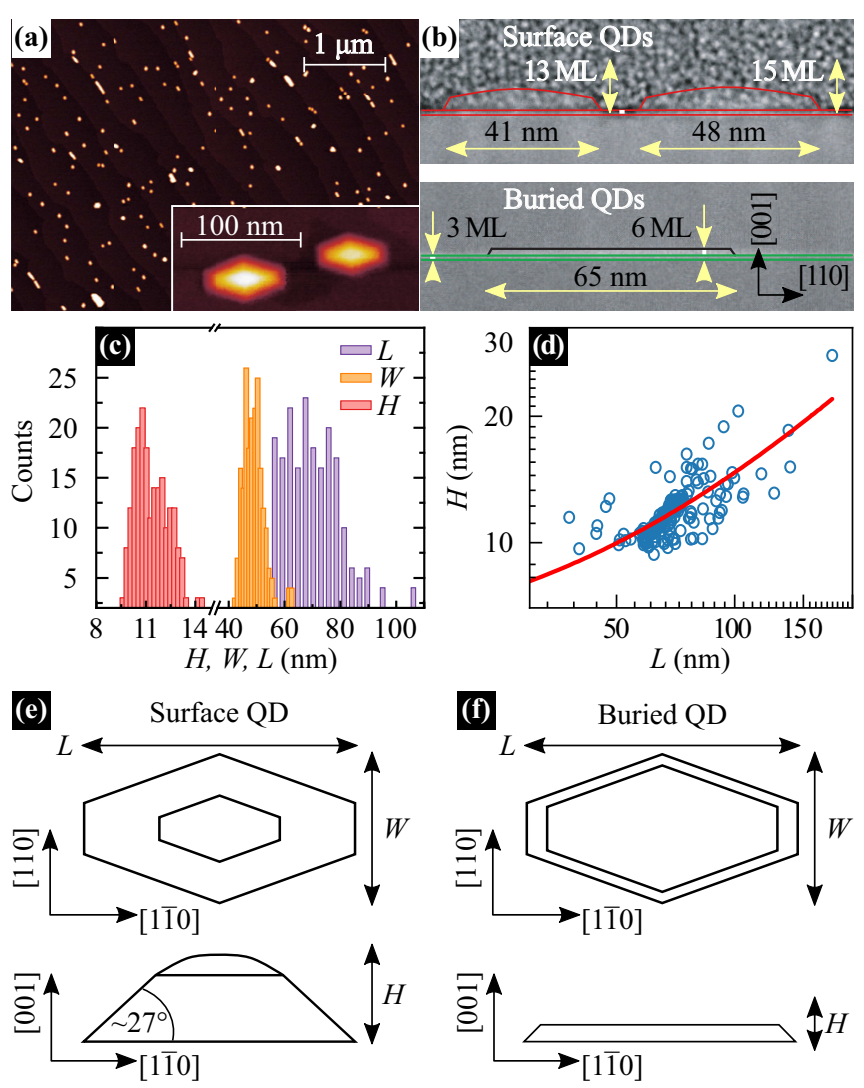

FIG. 1. (a) AFM image of $5 \times 3.5 \mu \mathrm{m}^{2}$ sample area with surface QDs. (Inset) Magnified part revealing geometry of single QDs. (b) TEM image of the sample cross-section with surface and buried QDs. (c) Histograms of width $W$, length $L$, and height $H$ of surface QDs based on the AFM data. (d) Dependence of surface QDs height on their in-plane size based on AFM data (symbols) with a linear trend (line). (e) and (f) Assumed shapes for the surface and buried QDs, respectively.

dimensions are similar to those of their buried counterparts, which are responsible for the strong optical response.

A high-angle annular dark-field scanning transmission electron microscopy (HAADF STEM) image of the structure is presented in Fig. 1(b), providing a cross-sectional view

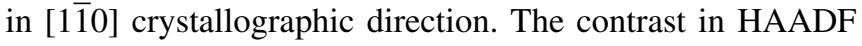
STEM images is primarily related to the atomic number, and can thus be used directly to measure the dimensions of the QDs, as the contrast arising from the strain is negligible here [23]. The upper panel reveals surface QDs, while the bottom one shows a single buried dot. Although some similarities between both types of dots are discernible, significant differences between them can also be observed. Both types of dots are of similar size in the growth plane. However, the height of buried QDs is much smaller than that of the surface ones. Additionally, the buried ones are flat on top [Fig. 1(f)], in contrast to dome-shaped surface dots [Fig. 1(e)]. These differences most probably result from longer growth interruption after the nucleation of the surface QDs. In this case, the material may be partially redistributed from the WL to the top of QDs, driven by strain relaxation. However, we can assume that the lateral sizes of surface dots are the same as of buried dots. The buried QD shown protrudes by $6 \mathrm{ML}$ from the WL top surface. We estimate that QDs are of pure InAs, based on the lattice spacing analysis of the STEM images available in Ref. [21].

\section{B. Optical experiments}

For time-integrated PL, photoluminescence excitation (PLE), and time-resolved PL (TRPL) experiments the structure was held in a helium closed-cycle refrigerator allowing for control of temperature in the range of 10-300 K. The sample was excited by a train of $\sim 2$-ps-long pulses generated by the optical parametric oscillator (OPO) providing a photon energy in the range of $0.9-1.2 \mathrm{eV}$. The pulse generation frequency was either $76 \mathrm{MHz}$ or lower. The light emitted from the sample was collected in a standard lens-based farfield optical setup and dispersed by a 0.3 -m-focal-length monochromator. Time-integrated PL was measured in a wide spectral range of $0.5-1.1 \mathrm{eV}$ via the lock-in technique at the reference modulation frequency of $2 \mathrm{kHz}$, using a thermoelectrically cooled InGaAs-based single-channel detector. The same experimental setup was employed for the PLE measurements. In this case, the monochromator was fixed to a certain detection energy $\left(E_{\mathrm{det}}\right)$ while the structure was excited by scanning the photon energy across the OPO's spectral range. The TRPL was measured by a time-correlated single photon counting method. Photons were spectrally filtered by the monochromator and subsequently directed onto the $\mathrm{NbN}$ superconducting detector. The multichannel event timer was synchronized to the pulse train to produce photon event statistics. The overall temporal resolution of the TRPL setup was $\sim 80$ ps.

For the PL with high spatial resolution ( $\mu \mathrm{PL})$ the sample was kept in a He-flow cryostat allowing for control of the sample temperature in the range of 5-300 K. Sample was excited by the continuous wave $(\mathrm{CW})$ semiconductor laser, and the emission was collected by a near-infrared-optimized microscope objective with high numerical aperture of NA = 0.4 . The $\mu \mathrm{PL}$ signal was spectrally analyzed by a $1-\mathrm{m}$-focallength monochromator and registered by a liquid-nitrogencooled InGaAs-based linear detector operating in the spectral range from $1.24 \mathrm{eV}$ down to $0.75 \mathrm{eV}$. Polarization properties of emitted light were analyzed by rotating the half-wave retarder mounted before a fixed high-contrast-ratio $\left(10^{6}: 1\right)$ linear polarizer placed in front of the monochromator's entrance.

\section{THEORY}

In this section, we describe the theoretical framework used to model investigated QDs and their electronic and optical properties.

\section{A. Modeling of QDs}

The modeling of nanostructures in question was preceded by evaluation of our initial assumption on their height varying by single MLs and by establishing the thickness of the WL. For this, numerical calculations within the eight-band $\boldsymbol{k} \cdot \boldsymbol{p}$ method with use of the commercially available nextnano software have been performed [24]. As a result, via comparison 
with experimental data, a WL thickness of 3 MLs has been determined.

To model the buried QDs we have used the available structural data presented in Sec. II. While the in-plane shape is not well-established, we followed the premise derived from surface dots and assumed an elongated hexagonal base with aspect ratio of $L / W=5 / 4$. The height, based on the crosssectional data, is of a few MLs of InAs. Additionally, from the spacing of PL peaks we formed an assumption that the dots are of height equal to consecutive integer numbers of MLs. Thus, we have modeled a series of such QDs protruding by $N$ MLs above the WL of 3-ML thickness, for $N=1-9$. For each height, we simulated QDs with in-plane sizes varied uniformly from $15 \mathrm{~nm} \times 12 \mathrm{~nm}$ up to $60 \mathrm{~nm} \times 48 \mathrm{~nm}$ in seven linear steps. Again following the properties of surface QDs, we set the inclination of QD side walls to $27^{\circ}$, which is however of little relevance in view of their small height. Regarding the material composition, we assumed that QDs and WL are composed of pure InAs.

\section{B. Single-particle states and complexes}

The lattice mismatch between InAs and InP is only approximately $3 \%$, nonetheless we have calculated the structural strain within the standard continuous elasticity theory via minimization of elastic energy. Next, piezoelectric field resulting from the presence of shear strain in a noncentrosymmetric material has been calculated up to second-order terms in strain-tensor elements. With this, to calculate the electron and hole eigenstates, and then states of their complexes, we have used a numerical implementation [28] of the eight-band $\boldsymbol{k} \cdot \boldsymbol{p}$ theory with envelope-function approximation $[29,30]$. To fix the spin configuration, a weak $(20 \mathrm{mT})$ magnetic field was used. The Dresselhaus spin-orbit interaction enters via perturbative terms in Hamiltonian blocks that couple the conduction band to valence bands, while the impact of structural strain is included via the standard Bir-Pikus Hamiltonian [31]. The explicit form of the Hamiltonian and description of the numerical implementation may be found in Ref. [32], while the material parameters used are given in Table I along with their sources. Numerical diagonalization yields the conduction- and valence-band electron eigenstates in the form of pseudospinors with components holding the envelope functions for each of the subbands. Hole states were obtained by application of time reversal operation to valence-band electron states.

Next, the calculated electron and hole single-particle states have been used to calculate the neutral- and charged-exciton, as well as biexciton states in the configuration-interaction approach. To this end, electron-hole Coulomb and phenomenological anisotropic exchange interactions have been diagonalized in the basis of $40 \times 40$ electron-hole configurations, which yielded carrier-complex states expanded in the configuration-space basis. Finally, for each of the states the coupling to light has been evaluated in the dipole approximation, and the resultant dipole moments were used to calculate radiative lifetimes of carrier-complex states [33].
TABLE I. Material parameters used in the modeling of nanostructures and calculation of single-particle and exciton states.

\begin{tabular}{lcccc}
\hline \hline Parameter & Unit & InAs & InP & Source \\
\hline$a$ & $\AA$ & 6.06 & 5.87 & {$[25]$} \\
$E_{\mathrm{g}}$ & $\mathrm{eV}$ & 0.417 & 1.42 & {$[25]$} \\
$\mathrm{VBO}$ & $\mathrm{eV}$ & -0.59 & -0.94 & {$[25]$} \\
$E_{\mathrm{p}}$ & $\mathrm{eV}$ & 21.5 & 20.7 & {$[25]$} \\
$m_{\mathrm{e}}^{*}$ & & 0.0229 & 0.0803 & {$[26]$} \\
$\Delta$ & $\mathrm{eV}$ & 0.39 & 0.11 & {$[25]$} \\
$\gamma_{1}$ & & 20.4 & 4.95 & {$[26]$} \\
$\gamma_{2}$ & & 8.3 & 1.65 & {$[26]$} \\
$\gamma_{3}$ & & 9.1 & 2.35 & {$[26]$} \\
$e_{14}$ & $\mathrm{C} / \mathrm{m}^{2}$ & -0.111 & 0.016 & {$[27]$} \\
$B_{114}$ & $\mathrm{C} / \mathrm{m}^{2}$ & -1.17 & -1.54 & {$[27]$} \\
$B_{124}$ & $\mathrm{C} / \mathrm{m}^{2}$ & -4.31 & -3.62 & {$[27]$} \\
$B_{156}$ & $\mathrm{C} / \mathrm{m}^{2}$ & -0.46 & -1.02 & {$[27]$} \\
$C_{\mathrm{k}}$ & $\mathrm{eV} \mathrm{A}$ & -0.0112 & -0.0144 & {$[26]$} \\
$a_{\mathrm{c}}$ & $\mathrm{eV}$ & -5.08 & -6.0 & {$[25]$} \\
$a_{\mathrm{v}}$ & $\mathrm{eV}$ & 1.0 & 0.6 & {$[25]$} \\
$b_{\mathrm{v}}$ & $\mathrm{eV}$ & -1.8 & -2 & {$[25]$} \\
$d_{\mathrm{v}}$ & $\mathrm{eV}$ & -3.6 & -5 & {$[25]$} \\
$c_{11}$ & $\mathrm{GPa}$ & 833 & 1011 & {$[25]$} \\
$c_{12}$ & $\mathrm{GPa}$ & 453 & 561 & {$[25]$} \\
$c_{44}$ & $\mathrm{GPa}$ & 396 & 456 & {$[25]$} \\
$\varepsilon_{\mathrm{r}}$ & & 14.6 & 12.4 & {$[26]$} \\
\hline \hline & & & & \\
\hline \hline
\end{tabular}

\section{RESULTS AND DISCUSSION}

In this section, we present both experimental and theoretical results. Their confrontation and mutual feedback are used as a base to discuss in detail the revealed properties of the system.

\section{A. Emission from ensemble of QDs}

We begin with presenting the results of PL measurements performed on the ensemble of QDs in question. These are presented in Fig. 2(a), where at $T=10 \mathrm{~K}$ a multimodal distribution of intensity with well-pronounced seven PL maxima (labeled A to G) is visible. The peaks spread across the energy range of $\sim 0.65-1.05 \mathrm{eV}$, and, notably, those labeled with $\mathrm{D}, \mathrm{E}$, and $\mathrm{F}$ coincide with the $\mathrm{S}, \mathrm{C}$, and $\mathrm{L}$ bands of the third telecom window, respectively. Combining this observation with knowledge on the growth process, we conjecture that each of the PL bands corresponds to emission from a distinct family of QDs. Such multi-peaked PL was previously observed for InAs/InP QDs grown via chemical beam epitaxy (CBE) [34,35], MBE [36,37], and MOVPE [38,39], as well as for InAs/GaAs QDs [40]. Based on our initial theoretical estimations, to form such a pattern of bands in emission, QDs have to be of height varying by a single InAs ML $(\approx 0.3 \mathrm{~nm})$, and each peak corresponds to a given QD height. Increase of temperature up to $T=300 \mathrm{~K}$ results in the PL pattern presented in Fig. 2(b), which still reflects the multimodal QD-height distribution. Thus, the confinement for carriers is strong enough, so that they are still localized, as it could be expected for QDs of size and composition assumed here.

To strengthen our interpretation of the ground-state nature of emission for all observed PL peaks, in Fig. 3, we plot 


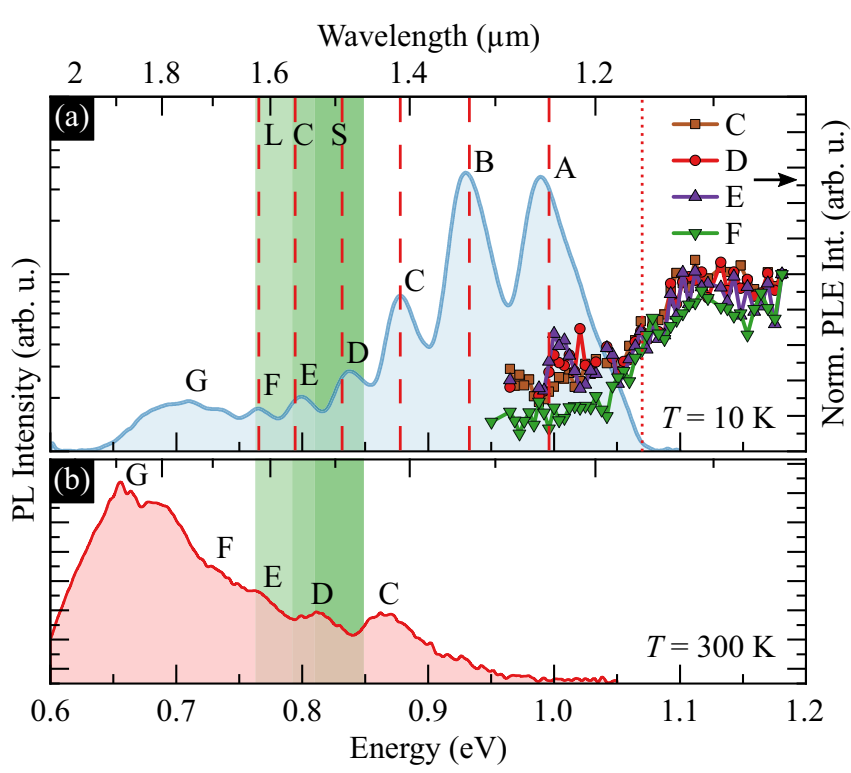

FIG. 2. (a) Low-temperature $(T=10 \mathrm{~K})$ PL spectrum (solid line, logarithmic scale) and PLE signals for the ensemble of QDs obtained for collection from lines C-F (symbols). Vertical dashed lines mark theoretically calculated exciton energies for modeled QDs, and dotted line marks the wetting layer absorption edge. Shaded areas represent the S (1460-1530nm), C (1530-1565nm) and L (1565-1625nm) bands of the third telecom window. (b) PL spectrum measured at $T=300 \mathrm{~K}$.

the excitation power-dependent PL spectra. The emission pattern does not change qualitatively over three orders of magnitude of the excitation powers [Fig. 3(a)], exhibiting the same multi-peak structure, with two peaks emitting at highest energies being always the brightest at low $T$. Furthermore, the dependence of the integrated PL intensity on the excitation power [Fig. 3(b)] for the all PL peaks is linear in the whole investigated power range (over four orders of magnitude), with none of peaks saturating. These observations, combined with temperature-dependent characteristics of PL (see Fig. 4), single QD studies and agreement of numerical calculations with experimental findings, presented in the following part of this article, prove the ground-state character of emission for all PL peaks.

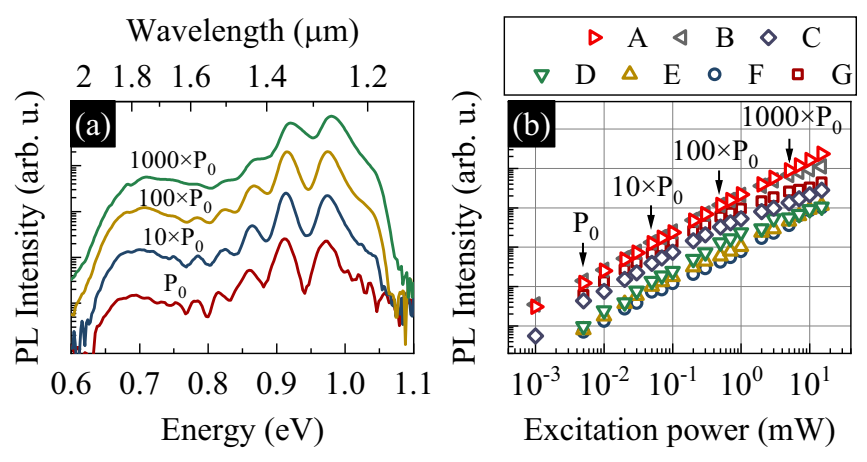

FIG. 3. (a) Power-dependent PL spectra at $T=10 \mathrm{~K}$ for excitation powers increasing by a factor of 10. (b) Integrated PL intensity for all peaks as a function of the excitation power.
Next, we focus on absorption properties of the dots, which are reflected in the PLE spectra. They were measured by registering the entire emission spectrum while scanning the excitation energy. Since no changes in the positions of PL peaks nor in their shapes were observed for QD families in question, each PLE trace presented in Fig. 2(a) is a plot of the PL peak intensity at a fixed detection energy versus the excitation energy. A strong absorption edge at about $1.07 \mathrm{eV}$ is present for all traces, which corresponds to the fundamental band gap of the WL. Moreover, since all traces are very similar, the WL beneath different QD families apparently has a similar thickness and chemical composition. Theoretical calculations of the InAs/InP WL fundamental gap as a function of the WL thickness $\left(d_{\mathrm{WL}}\right)$ and its further comparison to the measured absorption edge lead to $d_{\mathrm{WL}} \approx 3 \mathrm{ML}$. This value agrees with the WL thickness obtained from the crosssectional TEM presented in Fig. (1 b).

The quantitative agreement between positions of PL peaks and calculated exciton energies, visible in Fig. 2(a), has been achieved in the following way. As described in Sec. III, we have modeled a series of QDs with heights of consecutive integer numbers of InAs MLs and with varying in-plane size. This resulted in a smooth dependence of exciton ground-state energy on both varied QD dimensions. The latter could thus be interpolated via fitting of expected analytical dependence

$$
E(H, L) \simeq \sum_{i j} a_{i j} H^{-i} L^{-j},
$$

where $a_{i j}$ are the fit coefficients, whose nonnegative integer indices $i$ and $j$ do not exceed $i+j=2$. Next, we look for a smooth $L=f(H)$ dependence. From the surface-QD structural data we draw an assumption that it may be linear [see Fig. 1(d)], thus we plug $L=A H+B$ into Eq. (1), and fit the resultant one-variable function of $H$ to the dependence of PL-peak positions on the assumed $(N+3)$ ML height of corresponding QDs (a QD protrudes by $N$ MLs over the top of 3-ML-high WL). As a result, we obtain $L[\mathrm{~nm}]=$ $(3.2 \pm 0.5) N+(28 \pm 2)$, for which the final calculated energies are shown in Fig. 2(a), in a more than satisfactory agreement with positions of all peaks. The final series of sizes for modeled QDs established here is then used for calculation of other quantities presented in the following sections.

\section{B. Temperature-driven effects}

Here, we move to the temperature-driven processes in the system. In Fig. 4(a), we present the measured temperaturedependent PL spectra, where a strong redshift of emission is present, initially marked in Fig. 2(b). Moreover, an overall intensity drop of emitted light is observed. We attribute this PL quenching to redistribution of carriers between QDs, during which part of them escapes from the low-dimensional traps completely. Such processes may result from differences in confinement potential depth for distinct QD families. We explain the data similarly as in Ref. [41], with an assumption of transfer taking place via 2D wetting layer states. This transfer should involve thermal activation of carriers from QDs to the WL, their migration via WL states followed by their re-trapping with the highest probability by larger QDs with deeper confining potential, where the carriers finally 

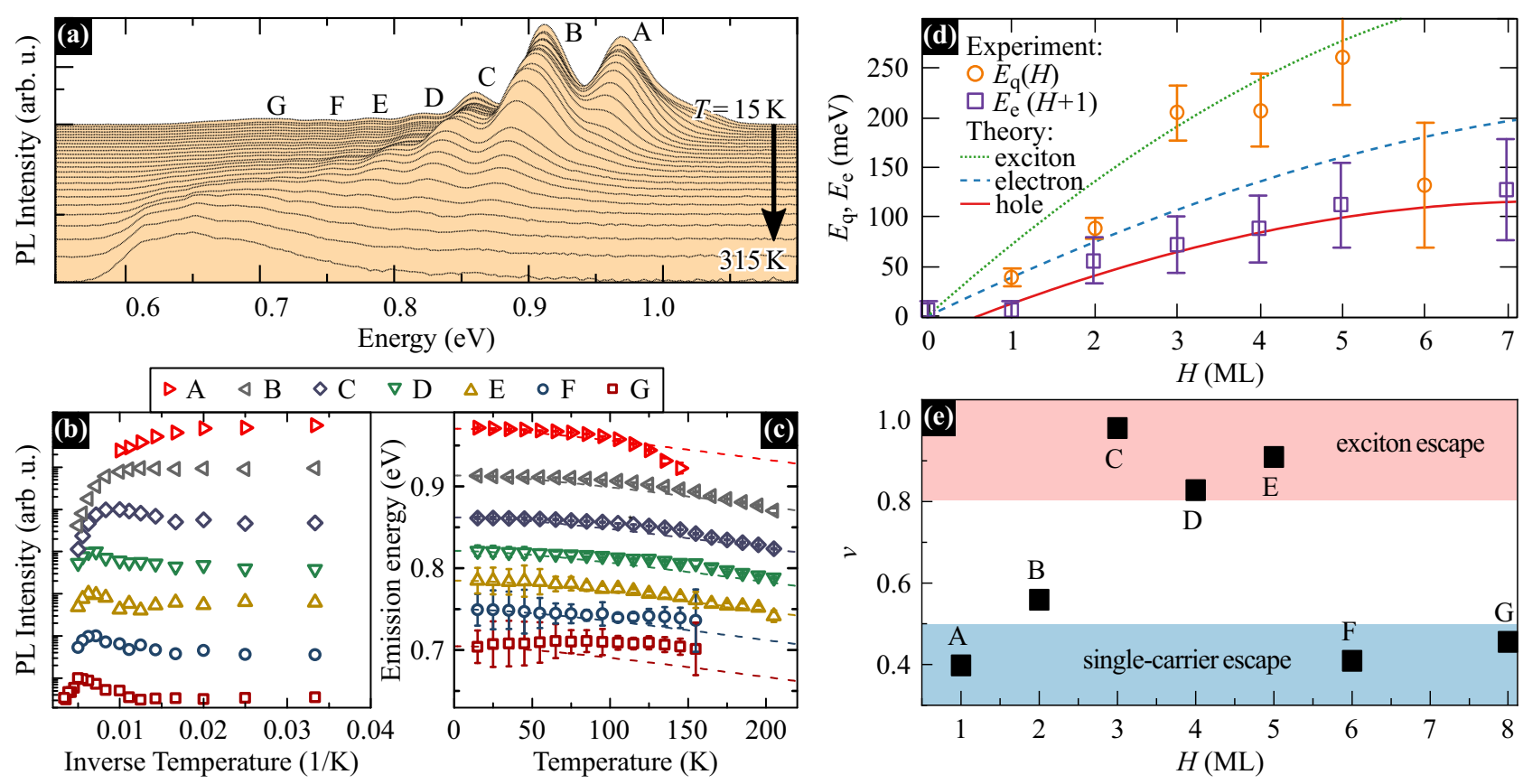

FIG. 4. Temperature dependence of PL emission from QDs. (a) Stacked PL spectra plotted in the Arrhenius form. (b) Temperature dependence of PL intensity for maxima A-G. (c) Thermal shift of emission energies (points) with Varshni lines: Eq. (2) (dashed lines). (d) Fitted activation energies for PL quench $E_{\mathrm{q}}$ (circles) and PL enhancement $E_{\mathrm{e}}$ (squares; shifted horizontally by one ML), and calculated energies for excitation to the WL ground state for excitons (dotted line), electrons (dashed), and holes (solid) in their ground states in a QD, versus QD height. (e) Analysis of phenomenological parameter $v$.

recombine. The activation-capture processes can be repeated many times before the radiative recombination. As a result, the temperature-dependent subtle interplay between activation and capture probabilities for each QD family is crucial for the relative PL intensities of peaks.

Note that other scenarios, such as the lateral tunneling of carriers or excitons can be excluded. In our sample, the average dot-to-dot variation is about $300 \mathrm{~nm}$, much larger than $\sim 10 \mathrm{~nm}$ required for this kind of lateral transport [42]. For the same reason, we exclude long-range dipole-dipole coupling $[43,44]$. Moreover, due to energy differences between QD ground states and the InP matrix, the lateral hopping of carriers seems improbable. Finally, we can exclude any significant impact of radiative recombination of excitons during the redistribution processes, as the characteristic times for carrier release from localized states and capture processes are both in the range of $\approx 20$ ps [41], i.e., two orders of magnitude faster than radiative recombination times observed by us for all QDs families (Fig. 5). Therefore excitons recombine mostly trapped in one of the QD families.

Such interpretation is supported by temperature dependence of intensities of each of the peaks plotted in the Arrhenius form in Fig. 4(b). Here, the initial enhancement of PL intensity from the lower-energy PL bands (C-F) is observed up to intermediate temperature range. These QD families correspond to deeper confinement for carriers. On the other hand, the highest-energy peaks exhibit only (band A) or mostly (B) a single process of PL quenching. This combination of observations illustrates how carriers are, on average, moved from lower to higher QDs. We note that the significant enhancement of PL intensities for QD families emitting in the third telecom window (families D-F) at abovecryogenic temperatures $(>77 \mathrm{~K})$ is particularly advantageous from the point of view of possible applications as sources of single photons operating at elevated temperatures.

In Fig. 4(c), we show how the PL intensity enhancement and quenching are accompanied by partly suppressed thermal redshift of the low-energy peaks $\mathrm{C}-\mathrm{G}$, and by enhanced one for the highest-energy band A. Nominally, for emission from a bulk material, a thermal redshift following the trend approximated by the Varshni relation is expected [45],

$$
E_{\mathrm{g}}(T)=E_{\mathrm{g}}(0)-\frac{\alpha T^{2}}{T+\beta},
$$

where $E_{\mathrm{g}}$ is the band-gap energy and parameters for InAs are $\alpha=2.76 \times 10^{-4} \mathrm{eV} / \mathrm{K}, \beta=93 \mathrm{~K}$. Corresponding curves are plotted with dashed lines. However, the Varshni formula takes into account only the thermal change of the semiconductor band gap. The minor blueshift from these predictions observed here is most probably related to the state filling effect: at higher temperature carriers are redistributed within each of the QD-family ensembles so that higher energy-emitting QDs get occupied, i. e., those smaller in-plane and thus characterized by weaker confinement. Therefore, the high-energy tails of QDs distributions become comparatively more optically active as the temperature increases.

For QDs with the shallowest confining potential (band A), emission energy initially follows the Varshni 

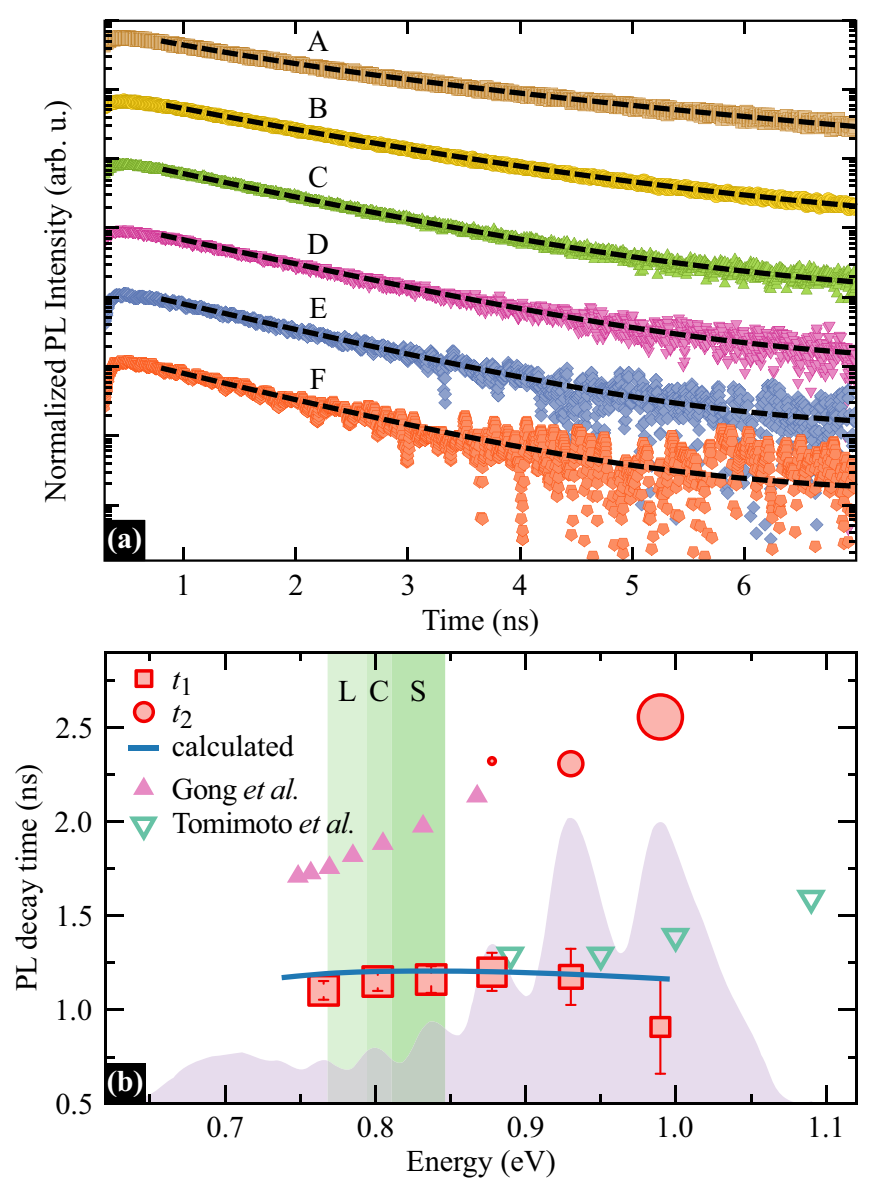

FIG. 5. (a) Time-resolved photoluminescence traces for all PL maxima (points) along with reconstructed curves (lines). (b) Experimentally obtained PL decay times (open symbols; values correspond to means of fitted lifetime distributions; point size marks the component amplitude) compared with calculated radiative exciton lifetimes (line) for QDs families. Triangles mark values obtained theoretically [51] (ム) and experimentally [52] ( $\nabla)$ for other InAs/InP QDs. relation, but around $120 \mathrm{~K}$ it considerably deviates due to fast depletion of high-energy QDs associated with strong PL quenching. For families B-E, the emission energy follows Eq. (2) within the entire temperature range. The different behavior for maximum $G$ exhibiting almost no energy shift may indicate that structures forming this peak do not form QD family analogous to QDs A-F, whose well-defined central emission energies shift spectrally. This interpretation is supported by very broad emission from this maximum (see Fig. 2). Instead, the maximum $G$ may be attributed to large material islands where the size, and hence emission energy, change quasi-continuously. Additionally, we may expect there a high density of structural defects due to the onset of strain relaxation, resulting in dislocations [46].

To verify these initial qualitative speculations, PL intensity data for peaks $\mathrm{A}$ and $\mathrm{B}$ was fitted with a formula taking into account one activation process of energy $E_{\mathrm{q}}$ and relative rate $B_{\mathrm{q}}[47]$ :

$$
I(T)=\frac{I_{0}}{1+B_{\mathrm{q}} \exp \left(-\frac{E_{\mathrm{q}}}{k_{\mathrm{B}} T}\right)},
$$

where $I_{0}$ is the PL intensity for $T \rightarrow 0$.

For emitters from families $\mathrm{C}-\mathrm{G}$, this analysis has to be extended to account for temperature-driven supply of carriers. Their recapturing and resulting PL enhancement is described by a characteristic energy $E_{\mathrm{e}}$ corresponding to release of carriers from their reservoir, and act as another source of occupation (apart from optical excitation) for the given bright state. Thus, we modify Eq. (3) in a straightforward manner by adding a state to the set of source kinetic equations from Ref. [47], on the same footage as the given emitting state has been introduced, but with different activation energy to the common higher state (here WL). With this a lengthy but simple to use formula accounting for exchange of carriers through the WL is obtained,

$$
I(T)=\frac{U P\left[R+U \exp \left(-\frac{E_{2}}{k_{\mathrm{B}} T}\right)\right]}{R U\left(U+R_{2}\right)\left[\exp \left(-\frac{E_{1}}{k_{\mathrm{B}} T}\right)+\exp \left(-\frac{E_{2}}{k_{\mathrm{B}} T}\right)\right]+U^{2} R_{2} \exp \left(-\frac{E_{1}+E_{2}}{k_{\mathrm{B}} T}\right)+R^{2}\left(2 U+R_{2}\right)},
$$

where $P$ is the pumping rate, $U$ is a common relaxation rate from the WL to both radiative states considered, $R$ and $R_{2}$ are the radiative and nonradiative loss rates also assumed equal for both states, while $E_{1}$ and $E_{2}$ are their energy differences from the WL ground state. Note that arbitrary changes made via educated guess in Eq. (3) that may be found in literature may lead to spurious values of extracted activation energies, if the form of modification does not comply with the nature of reservoir and its coupling to the given state. Activation energies for PL quenching and enhancement extracted in the aforementioned way are presented in Fig. 4(d) (points) versus the height given in the number of monolayers assigned to each of the peaks. Additionally, we plot the theoretically calculated energies needed for excitation of the electron, the hole, and the whole exciton (all in their ground states, trapped in a QD) to the WL ground state, taking the Coulomb coupling energies into account (lines). The results are obtained for the set of modeled QDs described above that led to agreement of exciton energies with maxima of PL bands. Comparing values of $E_{\mathrm{q}}$ to theoretical lines, we notice that also in this aspect peaks A and B behave differently than the few next ones. For these two, the activation energy related to quenching fits well to the value needed for escape of the electron from QD ground state. Then, for peaks C-E, we rather deal with an escape of whole excitons to the WL ground state. Another change of behavior is present for the lowest-energy peaks, where a value between electron and hole escape is found, which possibly means that both these processes take place 
with similar probability. To understand the source of carriers that feed the thermally enhanced emission in the moderate temperature regime, we plot the values of $E_{\mathrm{e}}$ extracted via fitting, but we shift them by one ML with respect to the value for which they were observed. This helps us to notice that possibly the most effective redistribution takes place between QDs with $1 \mathrm{ML}$ difference in height, i. e., that N-ML-high QDs are fed mostly by carriers that escape from QDs that are $(N-1)$-ML-high. Additionally, according to theoretical curves, this process is dominated by holes, which means that, while both carriers escape QDs, it is more probable for holes to be recaptured by another QD. This is intuitively in line with their higher effective mass and lower mobility. Thus, the assumption about migration of carriers between QDs from different families through the WL proves to be reasonable.

The dominant PL quenching mechanisms assessed via fitting are also in agreement with those determined by calculation of the phenomenological parameter

$$
v_{i}=\frac{E_{\mathrm{q}}^{(i)}}{\Delta E_{i}},
$$

where $i$ labels QD families, and $\Delta E_{i}$ stands for the difference between transition energies in the QDs and in the WL, $E_{\mathrm{WL}}$. From PLE experiments we take $E_{\mathrm{WL}}=1.07 \mathrm{eV}$ and obtain values presented in Fig. 4(e). Generally, $v<0.5$ indicates a dominant role of a single-carrier escape and in this case $E_{\mathrm{q}}$ corresponds to the lower of single-particle confinement energies (of either electron or hole) [48]. For $v=0.5$, we expect correlated escape of electron-hole pairs [49] and, finally, for $v \approx 1$ escape of excitons takes place [50].

\section{Photoluminescence dynamics}

The emission from all maxima has been investigated in the time domain by means of TRPL. Then, recorded PL decays were analyzed employing the PTI FELIXGX software by Photon Technology International that utilizes the maximal entropy method (MEM) [53,54], which among many applications has proved effective in multiexponential luminescence decay reconstruction $[55,56]$. It allows one to extract photoluminescence lifetimes without initial knowledge on the number of underlying processes, and thus decay components. Additionally, it does not introduce artificial features into the distribution of lifetimes, which are not supported by the data, if a reliable model of experimental noise is used. The analysis yielded the most probable distributions of PL decay constants $\tau_{i}$, which through the formula

$$
I(t)=I(0) \times \sum_{i=1}^{N} A_{i} \exp \left(-\frac{t}{\tau_{i}}\right)
$$

reconstruct the experimental curves with the level of uncertainty given by the amplitude of experimental noise. Here, $N$ is the number of components in the quasi-continuous distribution of lifetimes describing the decay, and $A_{i}$ are the corresponding amplitudes. The results of this analysis are presented in Fig. 5(a), where the TRPL traces collected for A-F luminescence peaks in the low excitation-power regime are shown along with the reconstructed curves. Via MEM reconstruction one obtains a distribution of times of nonzero width, which, if not really present in the system, represents the incomplete information carried by a noisy data. However, given a set of well-resolved peaks of amplitudes $A_{i}$, it is valid to treat their means as single representative values. Lifetimes presented with open squares and circles in Fig. 5(b) result from such an analysis. For the highest-energy peaks, we deal with two-exponential decays, where the shorter lifetime corresponds to recombination of excitons confined in a well-formed QD, while the longer one most probably comes from electron-hole pairs weakly bound on small WL width fluctuations. Amplitudes of each of decay components are marked with symbol size, and vanishing of the longer one with the number of MLs is present, which is reasonable, as no multiple-ML-wide fluctuations are expected.

Investigated QDs exhibit a dispersion of the $t_{1}$ PL lifetimes, which is different from what is expected and typically observed for more commonly studied InAs/GaAs QDs. In the latter, confinement characteristics for both types of carriers are comparable, with a weak tendency of hole wave function to leak into the barrier. Additionally, such QDs are typically of in-plane sizes not exceeding $\sim 30 \mathrm{~nm}$, which results in strong exciton confinement [57], characterized by a weak impact of electron-hole Coulomb interaction, energy of which is much smaller than single-particle level spacing. In this regime, the oscillator strength is simply defined by the overlap of electron and hole envelopes, which is close to 1 for a range of typical QD geometries. Thus, the observed dispersion of radiative lifetimes is caused mostly by the direct dependence of the latter on transition energy, which may be found in the dipole approximation as $\propto E^{-2}$.

Here we deal with radiative lifetimes of nearly no dispersion, which places investigated QDs in the middle between above-mentioned InAs/GaAs dots, and those grown in InP reported previously [51]. In the latter, the main source of radiative-lifetime dispersion for excitons has been found to come from the single-particle confinement characteristics, which are inversed in InP as compared to the GaAs matrix. In InP matrix, the electron is the particle that is more prone to leak into the barrier, as it experiences weaker confinement than holes, which is highly pronounced for flat QDs. The impact of electron leakage on exciton oscillator strength and lifetime in the InAs/InP QD system has been studied theoretically [51], and for completeness we plot the corresponding values with full triangles in Fig. 5(b). It was found that oscillator strength increases as electron wave function is increasingly better confined within the QD, i.e., for higher dots. For example, for a lens-shaped QD with diameter of $25 \mathrm{~nm}$ and height $H=2.5 \mathrm{~nm}$ only $53.5 \%$ probability of finding the electron inside the QD was reported, while for $H=5.5 \mathrm{~nm}$ this value increases to $81.2 \%$ [58]. Such decrease of lifetimes with decreasing energy was also observed experimentally [52] for InAs/InP QDs and is presented with open triangles in Fig. 5(b).

Due to very small height of QDs investigated here, especially those from families $\mathrm{A}$ and $\mathrm{B}$, we also deal with the reduction of oscillator strength caused by the weakly localized electron wave function. Contrarily, the hole is strongly localized within the dot, which reduces the overlap of their envelopes. Within the strong confinement limit this would lead to a direct reduction of coupling to light. With increasing 
QD height, the electron wave function tends to be more localized in the dot and its overlap with the hole becomes more complete, which reduces the observed PL lifetime. While this factor is common for all InAs/InP QDs, in the case of those studied here, it does not lead to expected inverted PL lifetime trend. Instead of this, a plateau is found, which means that another mechanism has to play a significant role.

We find it by investigating the details of exciton eigenstates comprised of Coulomb-correlated electron-hole configurations. Typically, any admixtures of higher bright singleparticle configurations into the exciton ground state result in the increase of oscillator strength. This purely quantum effect can be intuitively understood as the ability of the exciton to recombine simultaneously via each of the superposed electronhole pair states. For large QDs, in which level spacing for electrons and/or holes is low enough to be comparable with $\sim 20 \mathrm{meV}$ energy of their Coulomb interaction, this is the main source of lifetime reduction. While the dots considered here are flat, they are also relatively large in-plane. Additionally, which may seem counterintuitive, we find that reduced height results in stronger electron-hole Coulomb correlations, and as a result in larger admixtures of higher-energy configurations to the exciton ground state. For a fixed in-plane size of $L=$ $45 \mathrm{~nm}$, the admixture of higher electron-hole configurations is reduced from $16 \%$ to $5.5 \%$ when the height changes from 1 to 7 MLs. This may be understood, as the stronger the confinement along the growth axis, the more carrier wave functions are forced to penetrate the whole available in-plane area of the QD, which effectively increases the volume available for the exciton. This effect, pushing the dispersion of lifetimes in discussed QDs back towards the one known from InAs/GaAs dots underlies the observed approximately flat plot of lifetimes versus emission energy.

\section{Emission from single QDs}

To deepen the understanding of the system in question, and to check the applicability of QDs toward the single-photon emission from spatially well-resolved emitters, we have performed optical experiments on spatially and spectrally isolated QDs. For better spatial separation of single emitters, the sample was processed with electron-beam lithography and etched, leaving the array of mesa structures containing QDs. This enabled identification of exciton complexes and determination of their properties. An example of a single mesa is shown in the inset to Fig. 6.

We begin with presenting in Fig. 6 the results of $\mu \mathrm{PL}$ experiments performed on various mesas, which show intensive, well background-isolated emission lines. We attribute them to recombination of neutral exciton $(\mathrm{X})$ and biexciton (XX) complexes as well as to charged excitons (CX), each coming from a single QD. Spectral positions of lines are compared with the PL spectrum from the ensemble of dots, showing that they belong to the $\mathrm{D}, \mathrm{E}$, and $\mathrm{F}$ families of QDs emitting in the L, C, and $\mathrm{S}$ telecom bands, respectively. We note that lines within $\mathrm{L}$ band are at the edge of efficiency for InGaAs-based multichannel array detector utilized here $(\sim 1.6-1.65 \mu \mathrm{m})$, so single lines may also be present beyond this cut-off wavelength. Further identification of the emission lines shows that most of the dots emit due to recombination

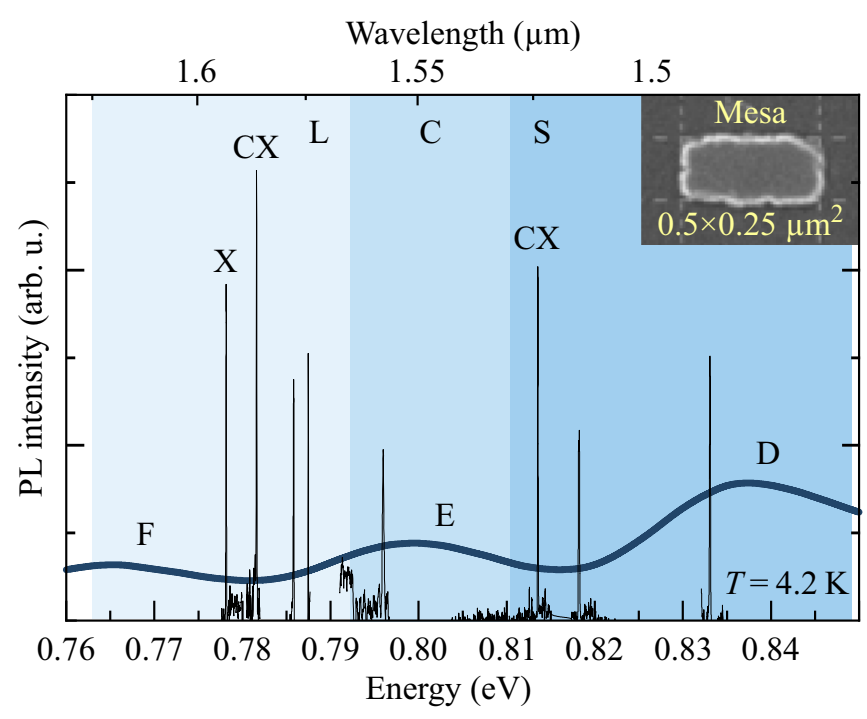

FIG. 6. Part of the $\mu$ PL spectrum from a mesa with QDs showing single emission lines observed at the third telecom window.

of charged exciton complexes (trions), and only a few of them show characteristics of the $\mathrm{X}$ and $\mathrm{XX}$ recombination. In this section, we present examples of $\mu \mathrm{PL}$ investigations for both neutral and charged complexes.

A pair of lines identified as the $\mathrm{XX}-\mathrm{X}$ recombination cascade is analyzed in Fig. 7. First, $\mu$ PL spectra taken under various excitation powers are shown in Fig. 7(a). From spectral positions of lines we determine the biexciton binding energy
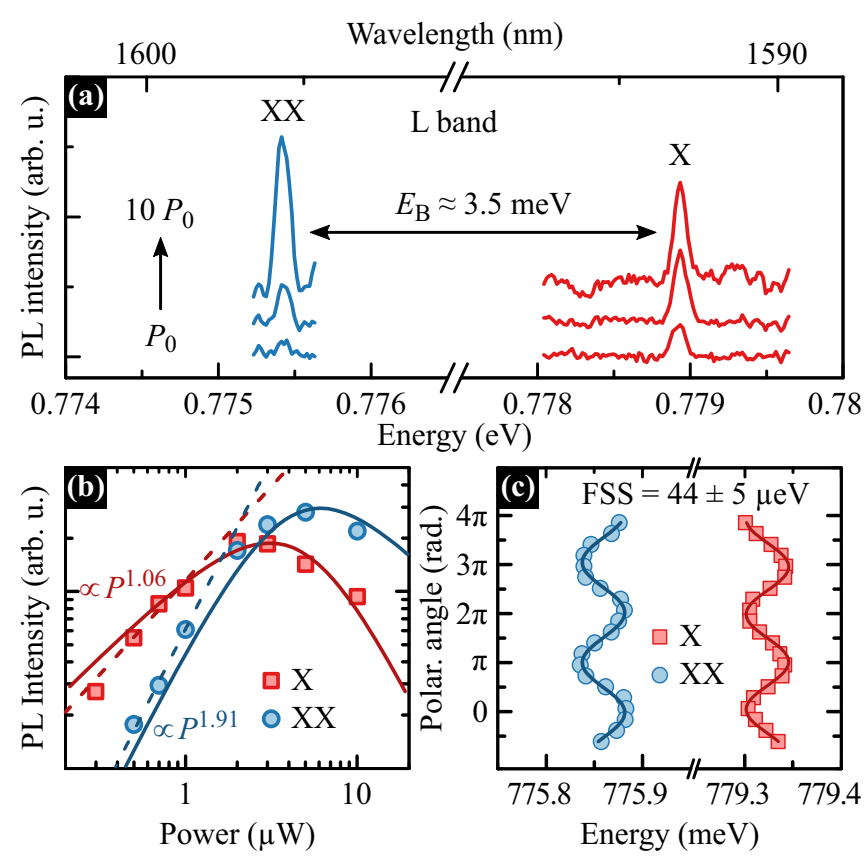

FIG. 7. Exciton (X) and biexciton (XX) emission at L band. Measured biexciton binding energy is $\sim 3.5 \mathrm{meV} . P_{0}=0.5 \mu \mathrm{W}$ (b) Power dependence of the $\mu \mathrm{PL}$ line intensities fitted with threelevel rate-equation model showing a correlation between $\mathrm{X}$ and $\mathrm{XX}$ lines. (c) Polarization-resolved $\mu \mathrm{PL}$ revealing exciton fine structure splitting of $E_{\mathrm{FSS}}=44 \pm 5 \mu \mathrm{eV}$. 


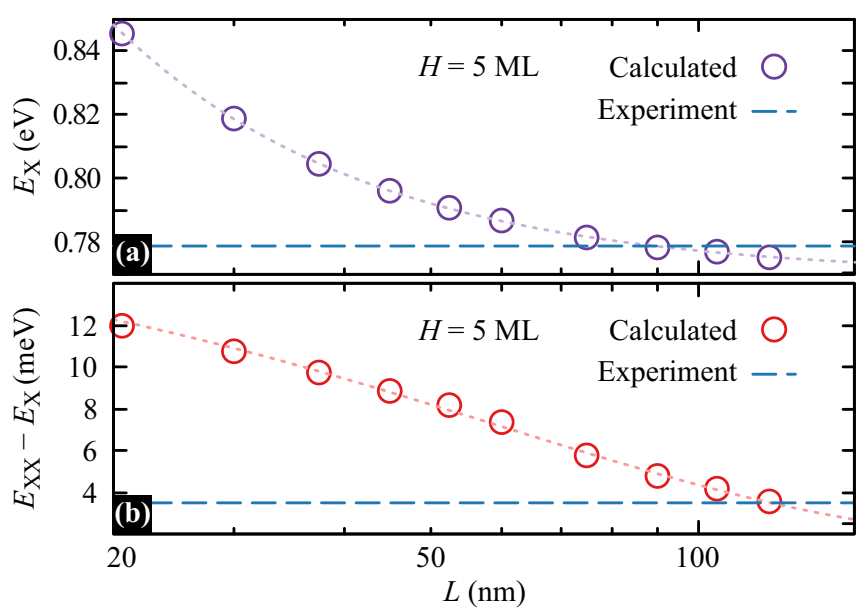

FIG. 8. (a) Dependence of calculated exciton energy on QD inplane size $L$ (circles) and the value of $\mathrm{X}$ line energy from experiment (dashed line). (b) Dependence of calculated biexciton binding energy on QD length (circles) and experimental value (dashed line). Dotted lines are to guide the eye.

of $E_{\mathrm{XX}}-E_{\mathrm{X}} \simeq 3.5 \mathrm{meV}$. The XX-X cascade was identified based on the power-dependent $\mu \mathrm{PL}$, shown in Fig. 7(b), and polarization-resolved $\mu \mathrm{PL}$ presented in Fig. 7(c). Both the three-level rate-equation model [59] [solid lines in Fig. 7(b)] and anti-phase of energy oscillations in Fig. 7(c) confirm correlation between observed lines. Additionally, in the lowpower regime, the power dependence of $\mathrm{X}\left(I_{\mathrm{X}}\right)$ and $\mathrm{XX}$ $\left(I_{\mathrm{XX}}\right)$ line intensities is well described with power laws: $I_{\mathrm{X}} \propto P^{1.06}$ and $I_{\mathrm{XX}} \propto P^{1.91}$, where $P$ is the excitation power. This is in a good agreement with expected approximately linear power dependence for $\mathrm{X}$ and quadratic for $\mathrm{XX}$. In the polarization-angle domain sinusoidal fits to the traces of emission energy yield the exciton fine structure splitting of $E_{\mathrm{FSS}}=44 \pm 5 \mu \mathrm{eV}$.

The X-line energy places the emitting QD in the lowenergy tail of the $\mathrm{E}$ family of QDs. Thus, properties different from those observed for the central part of the peak with the most typical QDs are expected. In particular, for a QD from this family to emit at such a long wavelength, the in-plane size has to be much larger than the average. To verify this, we use the results of a series of calculations for QDs with in-plane size varying up to $L=120 \mathrm{~nm}$. The resulting trend is plotted in Fig. 8(a) along with the measured energy of the $\mathrm{X}$ line (dashed line). As anticipated, the line is likely to come from a QD with large in-plane size close to $100 \mathrm{~nm}$. Next, we calculate also the biexciton biding energy in the same range of QD sizes, and plot the results in Fig. 8(b). Here, for $L=100 \mathrm{~nm}$, a binding energy of $\sim 4 \mathrm{meV}$ is found, which is close to the experimentally determined value. Thus, we conclude that the pair of lines is very likely to come from an atypically in-plane large QD.

Finally, in Fig. 9, we focus on charged complexes, optical signatures of which are found also within L, C, and S bands. Trions were identified based on close-to-linear power dependence of their intensity presented in Fig. 9(b), and the lack of any systematic variation in emission energy versus the angle of linear polarization of emission, as expected for
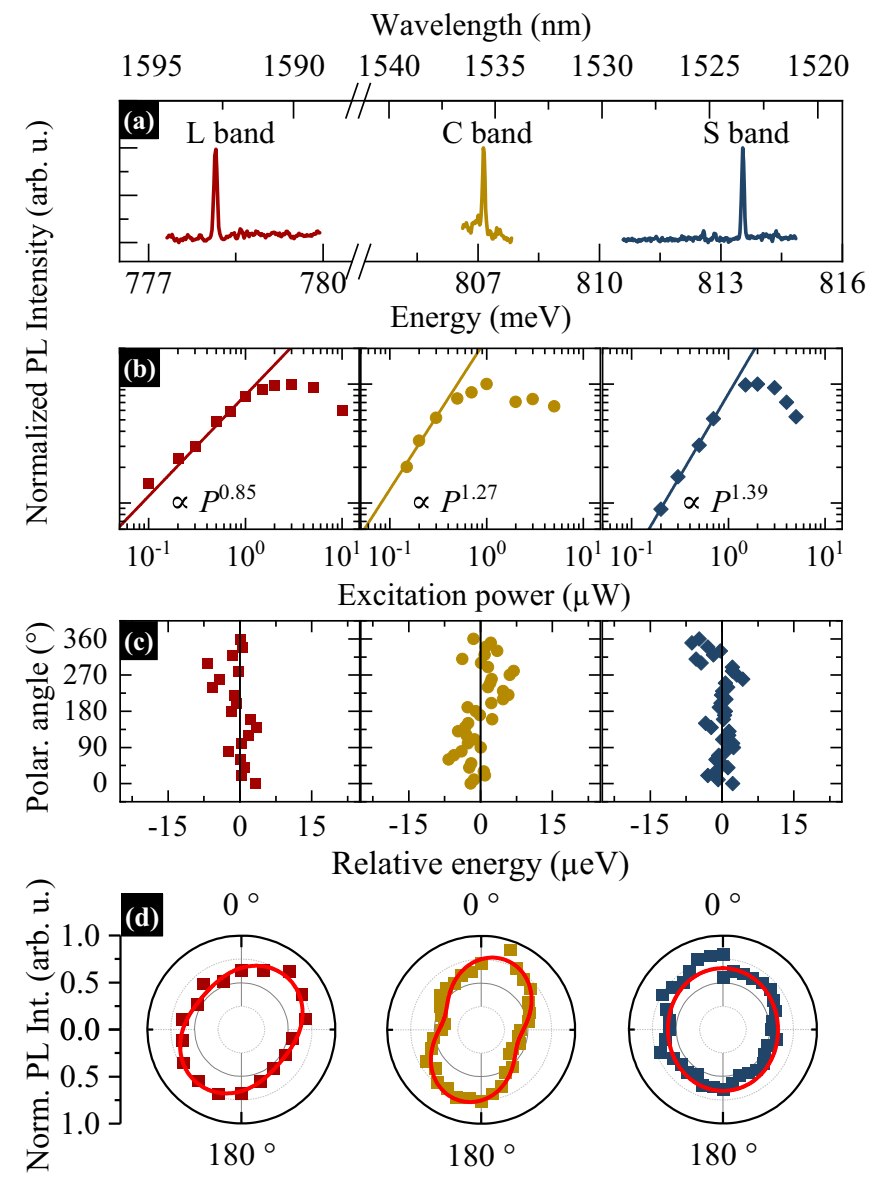

FIG. 9. Emission from charged excitons (CX) in the third telecom window. (a) $\mu \mathrm{PL}$ spectra. (b) $\mu \mathrm{PL}$ power dependence (points) with fitted power laws (lines). (c) Polarization-resolved $\mu \mathrm{PL}$ emission energy showing no fine structure splitting. (d) Anisotropy of polarization-resolved $\mu \mathrm{PL}$ intensity evidencing mixing of light-hole and heavy-hole states (lines are fits to the data).

spin singlet states [Fig. 9(c)]. Additionally, observed CX lines exhibit no phase correlation in polarization-resolved $\mu \mathrm{PL}$ to other nearby-lying lines within the biexciton binding energy range [such correlation is expected for neutral complexes, cf. Fig. 8(c)]. This spectral isolation strengthens their assignment as charged complexes.

In Fig. 9(d), we present the polarization-resolved $\mu \mathrm{PL}$ intensity plots for trions. The anisotropy is observed for all three CX lines as expected in asymmetric [60] and strained QDs [61] due to the mixing of light-hole and heavy-hole (LH-HH) subbands in these structures.

We estimate the degree of LH-HH mixing by fitting the function $I(\phi)=A\left(1+\rho_{L} \cos [2(\phi-\theta)]\right)$ [61] to the data in Fig. 9(d), where $I(\phi)$ is the PL intensity as a function of polarization angle, $A$ is a scaling factor, $\rho_{L}$ quantifies the degree of linear polarization, and $\theta$ is the offset from $0^{\circ}$, a value intrinsic for a given QD [61] and not necessarily corresponding to the elongation axis [60]. For investigated lines, we obtain values of $\rho_{L, L}=0.154 \pm 0.020, \rho_{L, C}=0.312 \pm 0.024$, and $\rho_{L, S}=$ $0.054 \pm 0.029$, for L-, C-, and S-band lines, respectively. The degree of linear polarization $\rho_{L}$ can be traced back to 

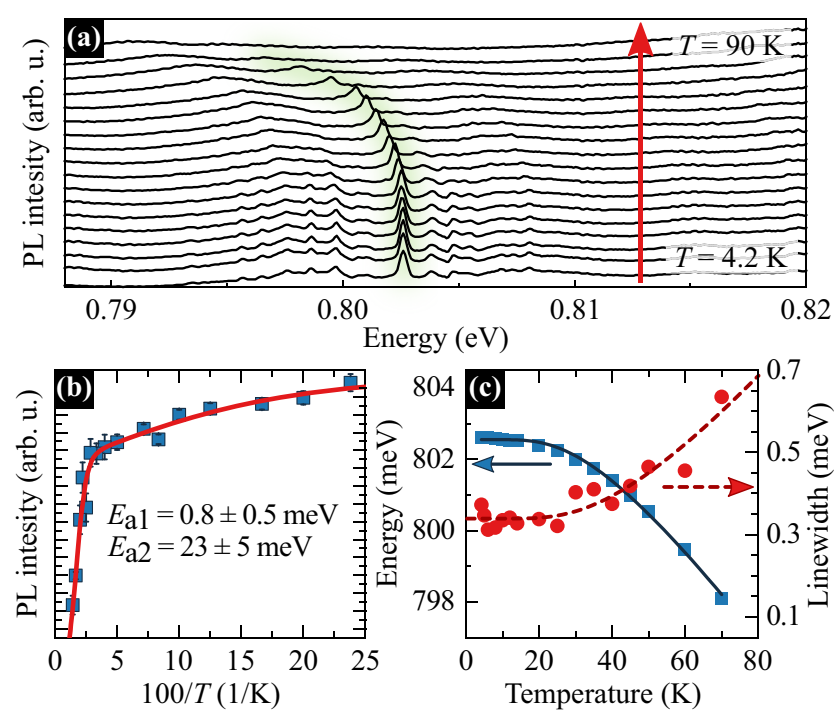

FIG. 10. Temperature dependence of emission for charged exciton from a 5-ML-high QD. (a) Stacked $\mu$ PL spectra. (b) Temperature dependence of the $\mu \mathrm{PL}$ intensity (points) with a fit (line). (c) Energy shift (squares) and linewidth broadening (circles) with fits (lines).

the degree of LH-HH mixing. Here, different values of $\rho_{L}$ evidence large dot-to-dot variation in this aspect.

For a CX line emitting in the $\mathrm{C}$ band we performed further $\mu \mathrm{PL}$ temperature-dependent studies reported in Fig. 10. The decreasing with temperature $\mu \mathrm{PL}$ signal was visible up to $T=$ $70 \mathrm{~K}$. The Arrhenius plot in Fig. 10(b) reveals two thermally activated processes. From fitting the data with Eq. (3) with two activation energies, we obtain values of $E_{\mathrm{a} 1}=1.0 \pm 0.5 \mathrm{meV}$ and $E_{\mathrm{a} 2}=23 \pm 5 \mathrm{meV}$. To interpret this result, it is vital to underline the difference in possible sources of PL quenching for the ensemble and single emitters. In the former case, the main source is related to thermal escape of carriers from the localization centers, such as QDs. Other thermal effects, like excitation of complexes to their higher orbital levels do not impact the integrated intensity from a PL band, as those excited states also emit there. On the other hand, if a single line is studied, excitation of a carrier complex to its higher state results in the emission occurring at a different wavelength that is not accounted for during the analysis of the emission intensity. As a result, quenching of a single $\mu \mathrm{PL}$ line reveals the relative energy of the first considerably bright excited state. The line investigated in Fig. 10 lies in the highenergy tail of the ensemble, thus most probably comes from a QD with a relatively small in-plane size. Calculation for a QD with $H=5 \mathrm{ML}$ and $L=28 \mathrm{~nm}$ yielded the lowest-energy bright excited CX states at $\Delta E=18.5$ and $18.7 \mathrm{meV}$ with radiative lifetimes of 2.7 and $2.1 \mathrm{~ns}$, respectively. Thus the energy $E_{\mathrm{a} 2}$ from experimental data may be attributed to the transfer of emission to a higher orbital state.

The thermal redshift of CX emission is presented with squares in Fig. 10(c) and fitted with formula accounting for phonon-mode occupation [62]. Increase of the latter enhances exciton-phonon interaction, influencing the chemical bonds and their energy and as a result it is the main factor leading to the band-gap renormalization [63,64]. Extracted average phonon energy is $E_{\mathrm{ph}, \mathrm{E}}=9.24 \pm 0.39 \mathrm{meV}$. We note that in contrast to Fig. 4(c), where we compared the thermal energy shifts of ensemble emission with Varshni curves, on the level of single emitters we can observe footprints of exciton-phonon interaction, therefore we use here more appropriate formula to explain the observed behavior.

The linewidth broadening $\Gamma(T)$ is presented in Fig. 10(c). The rather large initial value of $\Gamma(4.2 \mathrm{~K})=340 \mu \mathrm{eV}$ points at the crucial impact of defects or deep charge traps in the vicinity of a QD affecting significantly the emission properties. The broadening raises to $630 \mu \mathrm{eV}$ at $T=70 \mathrm{~K}$. The temperature dependence of linewidth was fitted with the formula that includes the contribution of thermally activated phonon sidebands to the zero-phonon line $[65,66]$,

$$
\Gamma(T)=\Gamma(4.2 \mathrm{~K})+a\left[\exp \left(\frac{E_{\mathrm{ph}, \Gamma}}{k_{B} T}\right)-1\right]^{-1},
$$

where $k_{B}$ is the Boltzmann constant, $a=1.5 \pm 1.1 \mathrm{meV}$, and $E_{\mathrm{ph}, \Gamma}=11.5 \pm 3.4 \mathrm{meV}$ is the average phonon energy. We note that average energies of phonons obtained from energy shift $E_{\mathrm{ph}, \mathrm{E}}=9.24 \pm 0.39 \mathrm{meV}$ and linewidth broadening $E_{\mathrm{ph}, \Gamma}=11.5 \pm 3.4 \mathrm{meV}$ agree well with one another and with previously reported value for InAs/InP stacked QDs [67].

\section{SUMMARY}

In conclusion, we have obtained InAs/InP QDs with low surface density grown by MOVPE and have studied their morphological and optical properties in detail, both experimentally and theoretically. The QDs are found to have families of different heights, from one to a few MLs of material above the WL, which is reflected in their emission with well-resolved multiple peaks covering a broad spectral range including the third window of silica-based optical fibers. In time-resolved spectroscopy, we observe dispersion of radiative lifetimes different from previously observed in InAs/InP QDs. While these typically show lifetimes increasing with emission energy in contrast to the opposite trend found in InAs/GaAs QDs, the dots under investigation show nearly no dispersion, which places them in between with respect to the electron-hole confinement parameters. With the aid of theoretical modeling, comprised of multiband $\boldsymbol{k} \cdot \boldsymbol{p}$ calculations of single-particle states and configuration-interaction method for neutral and charged excitons, we explain the observed dispersionless lifetimes as originating from the interplay of weak electron confinement (typical for InAs/InP) and strongly height-dependent exciton confinement regime. The latter is shown in the amount of higher single-particle eigenstates admixed to the exciton ground state, changing from 5.5\% for $7 \mathrm{ML}$ to $16 \%$ for $1 \mathrm{ML}$. Thus, the flatter the dot the weaker the exciton confinement regime, which may be understood in geometric terms as a result of higher effective in-plane size of a QD that is penetrated by carrier wave functions. At higher temperatures a redistribution of carriers among families of QDs takes place, in which we observe escape of both single particles and whole excitons resulting in PL quenching, as well as recapturing of holes by higher QDs leading to emission enhancement at moderate temperatures. Owing to the low surface density of QDs, we have observed $\mathrm{XX}-\mathrm{X}$ cascade and charged-exciton emission from single emitters persisting up to $T=70 \mathrm{~K}$. With theoretical 
modeling, we have reproduced experimental findings such as emission energies, radiative lifetimes, thermal activation energies for PL quenching and enhancement, and biexciton binding energy, which allowed for a comprehensive and detailed study of the system.

\section{ACKNOWLEDGMENTS}

We acknowledge support from the Villum Fonden via the NATEC Centre (8692), and YIP QUEENs project (VKR023442). P.H. acknowledges financial support from the Polish budgetary funds for science in 2018-2020 via the "Diamond Grant" program (Ministry for Science and
Higher Education, Grant No. DI 2017 011747). Numerical calculations have been carried out using resources provided by Wroclaw Centre for Networking and Supercomputing (http: //wcss.pl), Grant No. 203. We would like to thank Christian Schneider for mesa fabrication as part of the collaboration between Wrocław University of Science and Technology and the University of Würzburg within the International Academic Partnership project funded by the Polish National Agency for Academic Exchange, which also supported this work. We thank Krzysztof Gawarecki for sharing his implementation of the $\boldsymbol{k} \cdot \boldsymbol{p}$ method, as well as Janusz Andrzejewski and Grzegorz Sęk for helpful discussions.

P.H. and M.G. contributed equally to this work.
[1] I. Aharonovich, D. Englund, and M. Toth, Solid-state singlephoton emitters, Nat. Photonics 10, 631 (2016).

[2] P. Senellart, G. Solomon, and A. White, High-performance semiconductor quantum-dot single-photon sources, Nat. Nanotechnol. 12, 1026 (2017).

[3] L. Scarpelli, B. Lang, F. Masia, D. M. Beggs, E. A. Muljarov, A. B. Young, R. Oulton, M. Kamp, S. Höfling, C. Schneider, and W. Langbein, $99 \%$ beta factor and directional coupling of quantum dots to fast light in photonic crystal waveguides determined by spectral imaging, Phys. Rev. B 100, 035311 (2019).

[4] E. S. Semenova, R. Hostein, G. Patriarche, O. Mauguin, L. Largeau, I. Robert-Philip, A. Beveratos, and A. Lemaître, Metamorphic approach to single quantum dot emission at 1.55 $\mu \mathrm{m}$ on GaAs substrate, J. Appl. Phys. 103, 103533 (2008).

[5] F. Olbrich, J. Höschele, M. Müller, J. Kettler, S. L. Portalupi, M. Paul, M. Jetter, and P. Michler, Polarization-entangled photons from an InGaAs-based quantum dot emitting in the telecom C-band, Appl. Phys. Lett. 111, 133106 (2017).

[6] L. Seravalli, G. Trevisi, P. Frigeri, D. Rivas, G. MuñozMatutano, I. Suárez, B. Alén, J. Canet-Ferrer, and J. P. Martínez-Pastor, Single quantum dot emission at telecom wavelengths from metamorphic InAs/InGaAs nanostructures grown on GaAs substrates, Appl. Phys. Lett. 98, 173112 (2011).

[7] R. Kubota, T. Saiki, P. Regreny, A. Benamrouche, and M. Gendry, Low-density InAs quantum dots grown on $\operatorname{InP}(001)$ using solid-source molecular beam epitaxy with a post-growth annealing process, Jpn. J. Appl. Phys. 49, 041201 (2010).

[8] M. Benyoucef, M. Yacob, J. P. Reithmaier, J. Kettler, and P. Michler, Telecom-wavelength $(1.5 \mu \mathrm{m})$ single-photon emission from InP-based quantum dots, Appl. Phys. Lett. 103, 162101 (2013).

[9] M. Yacob, J. P. Reithmaier, and M. Benyoucef, Low-density InP-based quantum dots emitting around the $1.5 \mu \mathrm{m}$ telecom wavelength range, Appl. Phys. Lett. 104, 022113 (2014).

[10] K. Takemoto, Y. Sakuma, S. Hirose, T. Usuki, and N. Yokoyama, Observation of exciton transition in 1.3-1.55 $\mu \mathrm{m}$ band from single InAs/InP quantum dots in mesa structure, Jpn. J. Appl. Phys 43, L349 (2004).

[11] R. P. Leavitt and C. J. K. Richardson, Pathway to achieving circular InAs quantum dots directly on (100) InP and to tuning their emission wavelengths toward $1.55 \mu \mathrm{m}$, J. Vac. Sci. Technol. B 33, 051202 (2015).
[12] A. Musiał, P. Holewa, P. Wyborski, M. Syperek, A. Kors, J. P. Reithmaier, G. Sęk, and M. Benyoucef, High-purity triggered single-photon emission from symmetric single InAs/InP quantum dots around the telecom c-band window, Adv. Quantum Technol. 3, 1900082 (2019).

[13] T. Miyazawa, K. Takemoto, Y. Nambu, S. Miki, T. Yamashita, H. Terai, M. Fujiwara, M. Sasaki, Y. Sakuma, M. Takatsu, T. Yamamoto, and Y. Arakawa, Single-photon emission at $1.5 \mu \mathrm{m}$ from an InAs/InP quantum dot with highly suppressed multi-photon emission probabilities, Appl. Phys. Lett. 109, 132106 (2016).

[14] A. Musiał, P. Kaczmarkiewicz, G. Sęk, P. Podemski, P. Machnikowski, J. Misiewicz, S. Hein, S. Höfling, and A. Forchel, Carrier trapping and luminescence polarization in quantum dashes, Phys. Rev. B 85, 035314 (2012).

[15] M. Gawełczyk, M. Syperek, A. Maryński, P. Mrowiński, Ł. Dusanowski, K. Gawarecki, J. Misiewicz, A. Somers, J. P. Reithmaier, S. Höfling, and G. Sęk, Exciton lifetime and emission polarization dispersion in strongly in-plane asymmetric nanostructures, Phys. Rev. B 96, 245425 (2017).

[16] M. Gawełczyk, P. Wyborski, P. Podemski, J. P. Reithmaier, S. Höfling, and G. Sęk, Excited states of neutral and charged excitons in single strongly asymmetric InP-based nanostructures emitting in the telecom C band, Phys. Rev. B 100, 241304(R) (2019).

[17] M. Gawełczyk, Excitons in asymmetric nanostructures: Confinement regime, Acta Phys. Pol. A 134, 930 (2018).

[18] Ł. Dusanowski, P. Mrowiński, M. Syperek, J. Misiewicz, A. Somers, S. Höfling, J. P. Reithmaier, and G. Sęk, Confinement regime in self-assembled InAs/InAlGaAs/InP quantum dashes determined from exciton and biexciton recombination kinetics, Appl. Phys. Lett. 111, 253106 (2017).

[19] A. Sauerwald, T. Kümmell, G. Bacher, A. Somers, R. Schwertberger, J. P. Reithmaier, and A. Forchel, Size control of InAs quantum dashes, Appl. Phys. Lett. 86, 253112 (2005).

[20] K. Karrai and R. J. Warburton, Optical transmission and reflection spectroscopy of single quantum dots, Superlattices Microstruct. 33, 311 (2003).

[21] See Supplemental Material at http://link.aps.org/supplemental/ 10.1103/PhysRevB.101.195304 for the high-resolution STEM image with surface and buried QDs and for the map of fractional lattice-spacing changes, including Ref. [22]. 
[22] M. Hÿtch, E. Snoeck, and R. Kilaas, Quantitative measurement of displacement and strain fields from HREM micrographs, Ultramicroscopy 74, 131 (1998).

[23] S. Kadkhodazadeh, High resolution STEM of quantum dots and quantum wires, Micron 44, 75 (2013).

[24] S. Birner, T. Zibold, T. Andlauer, T. Kubis, M. Sabathil, A. Trellakis, and P. Vogl, nextnano: General purpose 3-D simulations, IEEE Trans. Electron Devices 54, 2137 (2007).

[25] I. Vurgaftman, J. R. Meyer, and L. R. Ram-Mohan, Band parameters for III-V compound semiconductors and their alloys, J. Appl. Phys. 89, 5815 (2001).

[26] R. Winkler, Spin-orbit Coupling Effects in Two-dimensional Electron and Hole Systems (Springer-Verlag GmbH, Berlin, Heidelberg, 2003).

[27] M. A. Caro, S. Schulz, and E. P. O'Reilly, Origin of nonlinear piezoelectricity in III-V semiconductors: Internal strain and bond ionicity from hybrid-functional density functional theory, Phys. Rev. B 91, 075203 (2015).

[28] K. Gawarecki, P. Machnikowski, and T. Kuhn, Electron states in a double quantum dot with broken axial symmetry, Phys. Rev. B 90, 085437 (2014).

[29] M. G. Burt, The justification for applying the effective-mass approximation to microstructures, J. Phys.: Condens. Matter 4, 6651 (1992).

[30] B. A. Foreman, Effective-mass Hamiltonian and boundary conditions for the valence bands of semiconductor microstructures, Phys. Rev. B 48, 4964 (1993).

[31] G. L. Bir, Symmetry and Strain-induced Effects in Semiconductors (Wiley, New York, 1974).

[32] K. Gawarecki, Spin-orbit coupling and magnetic-field dependence of carrier states in a self-assembled quantum dot, Phys. Rev. B 97, 235408 (2018).

[33] A. Thränhardt, C. Ell, G. Khitrova, and H. M. Gibbs, Relation between dipole moment and radiative lifetime in interface fluctuation quantum dots, Phys. Rev. B 65, 035327 (2002).

[34] A. Gustafsson, D. Hessman, L. Samuelson, J. F. Carlin, R. Houdré, and A. Rudra, Cathodoluminescence investigations of three-dimensional island formation in quantum wells, J. Cryst. Growth 147, 27 (1995).

[35] P. J. Poole, J. McCaffrey, R. L. Williams, J. Lefebvre, and D. Chithrani, Chemical beam epitaxy growth of self-assembled InAs/InP quantum dots, J. Vac. Sci. Technol. B 19, 1467 (2001).

[36] H. Folliot, S. Loualiche, B. Lambert, V. Drouot, and A. LeCorre, Effects of interface-layers composition and strain distribution on the optical transitions of InAs quantum dots on InP, Phys. Rev. B 58, 10700 (1998).

[37] Y. Berhane, M. O. Manasreh, H. Yang, and G. J. Salamo, Thermal annealing effect on the intersublevel transitions in InAs quantum dots, Appl. Phys. Lett. 78, 2196 (2001).

[38] Y. Sakuma, M. Takeguchi, K. Takemoto, S. Hirose, T. Usuki, and N. Yokoyama, Role of thin InP cap layer and anion exchange reaction on structural and optical properties of InAs quantum dots on InP (001), J. Vac. Sci. Technol. B 23, 1741 (2005).

[39] A. Lanacer, N. Shtinkov, P. Desjardins, R. A. Masut, and R. Leonelli, Optical emission from InAs/InP self-assembled quantum dots: evidence for As/P intermixing, Semicond. Sci. Technol. 22, 1282 (2007).

[40] F. Guffarth, R. Heitz, A. Schliwa, O. Stier, N. N. Ledentsov, A. R. Kovsh, V. M. Ustinov, and D. Bimberg, Strain engineering of self-organized InAs quantum dots, Phys. Rev. B 64, 085305 (2001).

[41] M. Syperek, M. Baranowski, G. Sęk, J. Misiewicz, A. Löffler, S. Höfling, S. Reitzenstein, M. Kamp, and A. Forchel, Impact of wetting-layer density of states on the carrier relaxation process in low indium content self-assembled (In, Ga)As/GaAs quantum dots, Phys. Rev. B 87, 125305 (2013).

[42] M. Bayer, P. Hawrylak, K. Hinzer, S. Fafard, M. Korkusiński, Z. R. Wasilewski, O. Stern, and A. Forchel, Coupling and entangling of quantum states in quantum dot molecules, Science 291, 451 (2001).

[43] T. Förster, Zwischenmolekulare energiewanderung und fluoreszenz, Ann. Phys. 437, 55 (1948).

[44] C. R. Kagan, C. B. Murray, and M. G. Bawendi, Long-range resonance transfer of electronic excitations in close-packed CdSe quantum-dot solids, Phys. Rev. B 54, 8633 (1996).

[45] Y. Varshni, Temperature dependence of the energy gap in semiconductors, Physica 34, 149 (1967).

[46] K. Yamaguchi, Self-formation of semiconductor quantum dots, in Handbook of Nano-Optics and Nanophotonics, edited by M. Ohtsu (Springer Berlin Heidelberg, Berlin, Heidelberg, 2013), pp. 809-843.

[47] J. D. Lambkin, D. J. Dunstan, K. P. Homewood, L. K. Howard, and M. T. Emeny, Thermal quenching of the photoluminescence of InGaAs/GaAs and InGaAs/AlGaAs strained-layer quantum wells, Appl. Phys. Lett. 57, 1986 (1990).

[48] G. Gélinas, A. Lanacer, R. Leonelli, R. A. Masut, and P. J. Poole, Carrier thermal escape in families of InAs/InP self-assembled quantum dots, Phys. Rev. B 81, 235426 (2010).

[49] W. Yang, R. R. Lowe-Webb, H. Lee, and P. C. Sercel, Effect of carrier emission and retrapping on luminescence time decays in InAs/GaAs quantum dots, Phys. Rev. B 56, 13314 (1997).

[50] S. Khatsevich, D. H. Rich, E.-T. Kim, and A. Madhukar, Cathodoluminescence imaging and spectroscopy of excited states in InAs self-assembled quantum dots, J. Appl. Phys. 97, 123520 (2005).

[51] M. Gong, W. Zhang, G. C. Guo, and L. He, Atomistic pseudopotential theory of optical properties of exciton complexes in InAs/InP quantum dots, Appl. Phys. Lett. 99, 231106 (2011).

[52] S. Tomimoto, A. Kurokawa, Y. Sakuma, T. Usuki, and Y. Masumoto, Radiative recombination of excitons in diskshaped InAs/InP quantum dots, Phys. Rev. B 76, 205317 (2007).

[53] E. T. Jaynes, Information theory and statistical mechanics, Phys. Rev. 106, 620 (1957).

[54] E. T. Jaynes, Information theory and statistical mechanics. II, Phys. Rev. 108, 171 (1957).

[55] J. C. Brochon and A. K. Livesey, Analyzing the distribution of decay constants in pulse-fluorimetry using the maximum entropy method, Biophys. J. 52, 693 (1987).

[56] A. T. N. Kumar, L. Zhu, J. F. Christian, A. A. Demidov, and P. M. Champion, On the rate distribution analysis of kinetic data using the maximum entropy method: applications to myoglobin relaxation on the nanosecond and femtosecond timescales, J. Phys. Chem. B 105, 7847 (2001).

[57] Al. L. Éfros and A. L. Éfros, Interband absorption of light in a semiconductor sphere, Fiz. Tekh. Poluprovodn. 5, 2191 (1982) [Sov. Phys. Semicond. 16, 772 (1982)]. 
[58] M. Gong, K. Duan, C.-F. Li, R. Magri, G. A. Narvaez, and L. He, Electronic structure of self-assembled InAs/InP quantum dots: Comparison with self-assembled InAs/GaAs quantum dots, Phys. Rev. B 77, 045326 (2008).

[59] G. Sęk, A. Musiał, P. Podemski, and J. Misiewicz, On the applicability of a few level rate equation model to the determination of exciton versus biexciton kinetics in quasizero-dimensional structures, J. Appl. Phys. 108, 033507 (2010).

[60] T. Belhadj, T. Amand, A. Kunold, C.-M. Simon, T. Kuroda, M. Abbarchi, T. Mano, K. Sakoda, S. Kunz, X. Marie, and B. Urbaszek, Impact of heavy hole-light hole coupling on optical selection rules in GaAs quantum dots, Appl. Phys. Lett. 97, 051111 (2010).

[61] A. V. Koudinov, I. A. Akimov, Y. G. Kusrayev, and F. Henneberger, Optical and magnetic anisotropies of the hole states in Stranski-Krastanov quantum dots, Phys. Rev. B 70, 241305(R) (2004).
[62] K. P. O'Donnell and X. Chen, Temperature dependence of semiconductor band gaps, Appl. Phys. Lett. 58, 2924 (1991).

[63] P. B. Allen and V. Heine, Theory of the temperature dependence of electronic band structures, J. Phys. C: Solid State Phys. 9, 2305 (1976).

[64] P. B. Allen and M. Cardona, Temperature dependence of the direct gap of Si and Ge, Phys. Rev. B 27, 4760 (1983).

[65] D. Gammon, E. S. Snow, B. V. Shanabrook, D. S. Katzer, and D. Park, Homogeneous linewidths in the optical spectrum of a single gallium arsenide quantum dot, Science 273, 87 (1996).

[66] G. Moody, M. E. Siemens, A. D. Bristow, X. Dai, D. Karaiskaj, A. S. Bracker, D. Gammon, and S. T. Cundiff, Exciton-exciton and exciton-phonon interactions in an interfacial GaAs quantum dot ensemble, Phys. Rev. B 83, 115324 (2011).

[67] J. Ishi-Hayase, K. Akahane, N. Yamamoto, M. Kujiraoka, K. Ema, and M. Sasaki, Negligible pure dephasing in InAs selfassembled quantum dots, Jpn. J. Appl. Phys. 46, 6352 (2007). 